\title{
CARACTERÍSTICAS DAS PEQUENAS E MÉDIAS EMPRESAS BRASILEIRAS QUE COOPERAM COM GRUPOS DE PESQUISA DE UNIVERSIDADES E INSTITUTOS PUBLICOS DE PESQUISA E DE SEUS RELACIONAMENTOS COOPERATIVOS
}

Vanessa Criscuolo Parreiras De Oliveira ${ }^{1}$

${ }^{1}$ Universidade Estadual de Campinas 


\section{CARACTERÍSTICAS DAS PEQUENAS E MÉDIAS EMPRESAS BRASILEIRAS QUE COOPERAM COM GRUPOS DE PESQUISA DE UNIVERSIDADES E INSTITUTOS PUBLICOS DE PESQUISA E DE SEUS RELACIONAMENTOS COOPERATIVOS}

\section{Resumo:}

O objetivo deste trabalho é investigar as características das pequenas e médias (PMEs) brasileiras que cooperam com grupos de pesquisa de universidades e institutos públicos de pesquisa e de seus relacionamentos cooperativos. Para cumprir com este objetivo são utilizadas informações do Diretório dos Grupos de Pesquisa no Brasil do Conselho Nacional de Desenvolvimento Científico e Tecnológico - DGP/CNPq (Censo 2010) e da Relação Anual de Informações Sociais (RAIS). Os resultados mostram que embora as PMEs que cooperam com universidades e institutos públicos de pesquisa sejam relativamente homogêneas em relação à intensidade da interação universidade-empresa (U-E), tais empresas são bastante heterogêneas, particularmente em relação ao número de empregados e ao número de empregados com nível superior ou mais (proxy adotada para a capacidade de absorção da empresa).

Palavras-chave: pequenas e médias empresas, cooperação, universidades, institutos públicos de pesquisa

\section{$1 \quad$ Introdução}

Em seus processos inovativos, as pequenas e médias empresas (PMEs) se deparam com vários problemas e obstáculos relacionados aos seus escassos recursos internos (ROTHWELL, 1989; FREEL, 2000; FONTANA et al., 2003; ZEVALLOS, 2003; MOLINA-YCAZA e SÁNCHEZ-RIOFRÍO, 2016, entre outros). Segundo Zevallos (2003), as condições nas quais as micro, pequenas e médias empresas (MPMEs) se desenvolvem na América Latina não lhes são particularmente favoráveis. Tais condições se relacionam, em grande parte, às suas competências endógenas (baixo grau de adoção tecnológica, pouca qualificação de seus trabalhadores e/ou do próprio empresário, fragilidade administrativa e baixa produtividade) e também a certas condições do ambiente (e institucionais), que as afetam em maior grau do que às grandes empresas. Molina-Ycaza e Sánchez-Riofrío (2016) acrescentam que os principais obstáculos para o desenvolvimento das MPMEs nestes países são o acesso ao financiamento e à tecnologia e a falta de recursos humanos especializados.

No Brasil, Chiarini et al. (2019) identificam, com base na Pesquisa de Inovação do Instituto Brasileiro de Geografia e Estatística (PINTEC/IBGE), que os obstáculos econômicos (e institucionais), tecnológicos e organizacionais são fatores que dificultam e impedem processos inovativos de forma semelhante em empresas 'inovadoras' e 'não inovadoras'. No entanto, o porte da empresa (seja ela inovadora ou não) importa. Isso é, quanto maior o tamanho da empresa, menos obstáculos ela enfrenta para inovar vis-à-vis as MPMEs.

O estabelecimento de laços de cooperação entre as MPMEs, através da promoção de clusters ou de alianças estratégicas, pode ser um instrumento importante para estimular o crescimento dessas empresas (ROVERE, 2001). Particularmente, as alianças estratégicas com 
universidades, centros tecnológicos e outras empresas do setor, que impliquem transferências de recursos tangíveis e intangíveis, podem constituir uma solução aos obstáculos com que essas firmas que se deparam (MOLINA-YCAZA e SÁNCHEZ-RIOFRÍO, 2016).

Estudos prévios, conceituais e empíricos, enfocaram os fatores direcionadores (drivers) dos relacionamentos cooperativos entre universidades e institutos públicos de pesquisa (IPPs) e empresas. É importante salientar que, apesar de haver uma ampla literatura que aborda os principais fatores direcionadores da interação U-E na perspectiva das firmas, há escassos trabalhos empíricos que enfocam esses relacionamentos cooperativos de PMEs. Frente a este contexto, este trabalho aborda os relacionamentos cooperativos de PMEs brasileiras e grupos de pesquisa de universidades e IPPs. Seu objetivo é investigar as características das PMEs que cooperam com grupos de pesquisa de universidades e IPPs e de seus relacionamentos cooperativos. Para cumprir com este objetivo são utilizadas informações do Diretório dos Grupos de Pesquisa no Brasil do Conselho Nacional de Desenvolvimento Científico e Tecnológico - DGP/CNPq (Censo 2010) e da Relação Anual de Informações Sociais (RAIS).

O texto está organizado em cinco seções, incluindo esta introdução. A segunda seção apresenta uma breve revisão da literatura empírica sobre a cooperação U-E, com foco em alguns dos principais direcionadores (drivers) do envolvimento das empresas com essa cooperação. A terceira seção aborda os procedimentos metodológicos adotados. A quarta seção expõe a discussão dos resultados de pesquisa, seguida pelas considerações finais na quinta seção.

\section{$2 \quad$ Referencial Teórico}

Estudos empíricos identificaram as principais variáveis que influenciam a propensão de as firmas cooperarem com universidades e IPPs. Da perspectiva daquelas, destacam-se fatores estruturais, comportamentais, geográficos e relacionados a políticas como os mais importantes impulsionadores da cooperação entre organizações públicas de pesquisa e a indústria (DE FUENTES e DUTRENIT, 2012).

Estudos que investigaram os fatores determinantes (drivers) da cooperação U-E, baseados em dados de países desenvolvidos, encontraram que o tamanho é positivamente relacionado à probabilidade de as firmas utilizarem o conhecimento de universidades e a cooperarem com elas (por exemplo, COHEN et al., 2002 e SANTORO e CHAKRABARTI, 2002, para os Estados Unidos); MOHNEN e HOAREAU (2003) para Alemanha, França, Irlanda e Espanha; FONTANA et al. (2003), para Dinamarca, França, Alemanha, Grécia, Itália, Holanda e Reino Unido; LAURSEN e SALTER (2004) para o Reino Unido; e CARDAMONE e PUPO (2015) para Alemanha, Itália, Espanha e Reino Unido. Os resultados destes trabalhos são consistentes com a hipótese de que a capacidade de as firmas utilizarem a pesquisa universitária aumenta com o tamanho da organização. Todavia, Torres et al. (2011) encontraram para o México que, quanto maiores as firmas, menor é a sua propensão a se engajarem em interações U-E. Os dados dos autores sugerem que pequenas firmas, provavelmente com atividades intensivas em $\mathrm{P} \& \mathrm{D}$, crescentemente procuram acessar o conhecimento de universidades e IPPs. Resultados similares para as firmas da Malásia permitiram a Rasiah e Govindaraju (2009) argumentarem que a relação entre o tamanho e a colaboração com universidades e IPPs não é óbvia. No Brasil, Bastos e Britto (2017) constataram, a partir da PINTEC/IBGE, que a variável porte tem importância no sentido de 
induzir a intensificação de relações cooperativas entre empresas inovativas nacionais e centros de produção científico-tecnológica.

A literatura sobre inovação salienta a existência de uma considerável variação interindustrial na propensão das firmas a utilizar universidades. Firmas em setores caracterizados por elevados níveis de investimento em P\&D e em outras atividades científicas e tecnológicas têm uma maior propensão a utilizar as universidades (LAURSEN e SALTER, 2004), de modo que pertencer a um setor científico aumenta a propensão do estabelecimento de colaborações diretas em inovação com universidades e laboratórios governamentais (MOHNEN e HOAREAU, 2003). Cohen et al. (2002) sugeriram que a pesquisa pública possui um impacto substancial sobre a $\mathrm{P} \& \mathrm{D}$ industrial em umas poucas indústrias, particularmente a farmacêutica, e é geralmente importante ao longo de um amplo segmento do setor manufatureiro. Schartinger et al. (2002) encontraram no grupo de setores com elevadas taxas de $\mathrm{P} \& \mathrm{D}$ que somente os setores de $\mathrm{P} \& \mathrm{D}$, indústria química, manufatura de instrumentos e a produção de veículos encontram-se ente os dez (10) setores com as maiores intensidades de interação U-E. Intensidades elevadas de interação podem ser observadas em setores manufatureiros mais orientados a recursos, tais como produção de energia, manufatura de metais básicos, manufatura de papel, construção e agricultura. No setor de serviços, as intensidades de interação U-E seguem um padrão, qual seja, elevadas intensidades nos serviços relacionados aos produtores, bancos, seguros e serviços de computação.

A habilidade de uma firma reconhecer o valor da nova informação externa, assimilá-la e aplicá-la a fins comerciais é crítica para suas capacidades inovativas (COHEN e LEVINTHAL, 1990). Sua capacidade de absorção é, em grande medida, uma função do nível de conhecimento anterior relacionado da firma. Cohen e Levinthal (1990) salientaram que a capacidade de absorção da firma refere-se não somente à aquisição ou assimilação de informação, mas também à habilidade para explorá-la (exploit). Portanto, a capacidade de absorção de uma firma não depende simplesmente da interface direta da organização com o ambiente externo. Ela depende também das transferências de conhecimento entre e dentro de subunidades. Segundo estes autores, a capacidade de absorção da firma depende dos indivíduos que permanecem na interface da firma e do ambiente externo ou na interface entre subunidades no interior da firma. A função de interface pode ser difundida entre indivíduos ou bastante centralizada. Quando a expertise da maioria dos indivíduos na organização difere consideravelmente daquelas dos atores externos que proporcionam informação útil, alguns membros dos grupos são prováveis de assumir papéis relativamente centralizados de gatekeeping" ou "boundary-spanning" (COHEN e LEVINTHAL, 1990).

Uma corrente da literatura investigou o impacto da capacidade de absorção da empresa sobre a cooperação U-E e identificou que a falta de capacidade de absorção pode constituir uma significativa barreira ao relacionamento cooperativo. Alguns autores salientaram a importância da capacidade de absorção das empresas como um fator influente na busca pela parceria U-E e na apropriação dos benefícios dessa interação. Uma alta capacidade de absorção - potencial e realizada - faz que com a firma esteja mais apta a transformar conhecimentos externos em novos produtos e processos produtivos, com efeitos importantes em seu desempenho inovativo (ROSA et al., 2018). A realização de investimentos em P\&D amplia a capacidade de absorção de conhecimentos da firma e, por essa razão, elas tendem a valorizar mais as universidades como fonte de informação (ALBUQUERQUE et al., 2005). As firmas com empregados 
altamente qualificados são as que mais colaboram com universidades (BRUNEEL et al., 2010; LAURSEN et al., 2011; DREJER e ØSTERGAARD, 2017).

Em relação aos determinantes das dimensões da capacidade de absorção, a capacidade de adquirir o conhecimento externo não está relacionada com a atividade de P\&D interna (TEIXEIRA et al., 2016), visto que se relaciona ao predomínio de trabalhadores com pósgraduação, que podem agir como gatekeepers do conhecimento, facilitando a identificação e a aquisição desse conhecimento na ausência de esforços mais estruturados de $\mathrm{P} \& \mathrm{D}$ na empresa.

Alguns autores exploraram o impacto da experiência prévia da firma em trabalhar em projetos de pesquisa com universidades sobre os relacionamentos cooperativo U-E. Segundo Cyert e Goodman (1997), esses relacionamentos constituem uma oportunidade para o aprendizado, que pode impactar o pensamento estratégico da organização, a cultura, as habilidades para resolução de problemas e a base de conhecimento. Para a corporação, a mudança organizacional relacionada ao relacionamento cooperativo com universidades pode se verificar em termos de novos produtos, políticas de aplicação ou práticas. Bruneel et al. (2010) observaram que trabalhar com universidades em projetos de pesquisa requer não somente que as firmas aprendam a trabalhar em fronteiras organizacionais (organizational boundaries), mas também requer que elas tenham ou possam construir as capacidades (capabilities) para colaborar com parceiros que operam em um sistema de incentivo diferente. A colaboração com um parceiro de universidade necessita que as firmas desenvolvam rotinas e práticas operacionais para gerir esta colaboração. Entretanto, uma vez que as rotinas e práticas tenham sido estabelecidas, elas são prováveis de serem refinadas e reutilizadas em colaborações subsequentes. Ainda segundo os autores, a experiência em colaboração poderia auxiliar também a reduzir as barreiras à colaboração relacionadas à transação.

Diferentes padrões de apoio financeiro também influenciam o estabelecimento e a frequência das colaborações U-E (DE FUENTES e DUTRENIT, 2012). Diversos estudos prévios investigaram o impacto das políticas de C\&T\&I e industrial sobre a cooperação U-E, os quais identificaram que o apoio governamental aumenta a incidência de exploração de conhecimento de universidades/laboratórios governamentais e o estabelecimento de colaborações diretas em inovação com eles (MOHNEN e HOAREAU, 2003; CARRIJO e BOTELHO, 2013; TORRES e BOTELHO, 2018).

\section{3}

\section{Metodologia}

Neste trabalho, a unidade de investigação é a firma interativa com os grupos de pesquisa do DGP/CNPq, conforme resposta do líder do grupo. As bases de informação utilizadas neste trabalho, que permitiram aferir aspectos da cooperação entre as PMEs e os grupos de pesquisa de universidades e IPPs no Brasil, são os dados do CNPq, coletados no DGP (Censo 2010) e na RAIS. A montagem da base do Censo 2010 pôde identificar a existência (ou não) de interações com firmas/instituições. As variáveis disponíveis na base referem-se: (1) aos grupos de pesquisa científicos; (2) às instituições parceiras com as quais interagem; e (3) aos tipos de relacionamento ${ }^{1}$.

\footnotetext{
${ }^{1}$ É importante ressaltar que trabalhos anteriores (por exemplo, RIGHI e RAPINI, 2011) apontaram uma subestimação das interações U-E declaradas pelos líderes dos grupos de pesquisa. Esse problema de subestimação permanece no Censo em questão (2010) e dever ser levado em consideração na análise dos resultados.
} 
As PMEs analisadas neste trabalho são aquelas que cooperaram, no Censo de 2010, com grupos de pesquisa das áreas de Engenharias e Ciências Agrárias. Estas são as duas áreas do conhecimento que apresentam o maior número de relacionamentos com o setor produtivo, conforme autores que investigaram as interações U-E no Brasil com base nos Censos 2004 (SUZIGAN et al., 2009; RIGHI e RAPINI, 2011) e 2008 (RAPINI et al, 2016).

Para a caracterização das instituições parceiras dos grupos de pesquisa dos campos científicos da Engenharia e das Ciências Agrárias foram adicionadas à base de dados, a partir do cruzamento dos CNPJs das empresas interativas da base do DGP/CNPq e da RAIS, as seguintes informações provenientes desta última base de dados: localização (Município, UF e $\mathrm{CEP}$ ), setor de atividade econômica, número de empregados e número de empregados com nível superior ou mais.

$\mathrm{Na}$ ausência de um critério para classificação das PMEs segundo a característica estrutural porte, comum a todos os setores de atividade econômica, foi adotada neste trabalho a estratificação da base de dados do DGP/CNPq e da RAIS pelo critério do Serviço Brasileiro de Apoio às Micro e Pequenas Empresas (SEBRAE) e do IBGE do porte do estabelecimento em função do número de pessoas ocupadas para os setores de atividade econômica da indústria e da construção ${ }^{2}$. Portanto, para a composição da base de dados referente às PMEs brasileiras interativas com grupos de pesquisa de universidades e IPPs, utilizou-se o recorte de CNPJs das instituições parceiras de grupos de pesquisa com até 499 empregados.

Aplicado o recorte de CNPJs com até 499 empregados na base de dados, a tabulação indicou um conjunto de 2049 instituições parceiras que interagiram com 1330 grupos de pesquisa do CNPq no Censo de 2010 (o equivalente a 66,90\% das instituições parceiras de grupos de pesquisa neste Censo).

Ademais, foram aplicados dois filtros à base de dados com o objetivo de refinar o critério do porte do estabelecimento em função do número de pessoas ocupadas. O primeiro se refere ao recorte dos dados das instituições parceiras a partir do "CNPJ Raiz", composto pelos oito primeiros números que formam o número de inscrição da empresa no Cadastro Nacional da Pessoa Jurídica (a "raiz", que identifica a empresa). Procurou-se, dessa forma, eliminar a diferenciação entre matriz e filial(is), representada pelos quatro dígitos seguintes, que identificam uma unidade de atuação da empresa (ou seja, um endereço de atividade da pessoa jurídica). Aplicado este filtro a todas as empresas da base, todas as informações referentes à instituição parceira passaram a abranger no "CNPJ raiz" as informações referentes a todos os estabelecimentos inscritos (filiais e matrizes). Todas as instituições parceiras de grupos de pesquisa do Censo 2010, cujos "CNPJs Raiz" corresponderam a um número de empregados igual ou superior a 500, foram eliminadas da base de dados.

Às empresas restantes na base de dados do DGP/CNPq foi aplicado um segundo filtro. Suas informações foram cruzadas com o Anuário Melhores e Maiores de 2010, da EXAME, no qual foi possível identificar as 1.000 maiores empresas do Brasil pelo critério do desempenho da receita líquida em dólares, excluídas as empresas do setor financeiro. Todas aquelas

\footnotetext{
2 Segundo este critério de classificação dos estabelecimentos segundo o porte nos setores industrial e de construção, as microempresas são aquelas que empregam até 19 pessoas; já as pequenas empresas são definidas como as que empregam de 20 a 99 pessoas. Por sua vez, os estabelecimentos com 100 ou mais empregados foram considerados como estabelecimentos de médias empresas e; empresas grandes, aquelas com 500 ou mais empregados (Ver "Nota Metodológica para Definição dos Números Básicos de MPE”, SEBRAE, 2006, citado por SEBRAE, 2013, p. 06).
} 
empresas da base de dados que apresentaram o número de empregados igual ou superior a 500 no referido Anuário foram eliminadas da base de PMEs interativas do DGP/CNPq.

Após a aplicação dos dois filtros acima referidos nas 2049 instituições parceiras dos grupos de pesquisa do CNPq encontram-se presentes na base de dados 1821 PMEs interativas. Foram também removidas da base de dados duas empresas que não apresentavam a informação "tipo de relação" para com um grupo de pesquisa. Dessa forma, os dados analisados neste trabalho referem-se às 1819 PMEs (ou 59,34\% das instituições parceiras dos grupos da base de dados do Censo de 2010) que tiveram algum tipo de relacionamento U-E com grupos de pesquisa de universidades e IPPs.

A partir da revisão de estudos empíricos e de alguns estudos de caso sobre relacionamentos cooperativos U-E foram definidos os principais fatores direcionadores (drivers) que podem influenciar o engajamento de PMEs em tais relacionamentos cooperativos. São eles: características estruturais e comportamentais das empresas interativas (isto é, o número de empregados; o setor de atividade econômica; a interação no Censo anterior do DGP/CNPq, tomada como proxy da experiência prévia em colaboração U-E; e o percentual de funcionários com nível superior ou mais, tomado como proxy da capacidade de absorção da firma). Ademais, são investigados fatores relacionados aos relacionamentos U-E de PMEs (número de interações e tipos de relacionamentos realizados pela firma com grupos de pesquisa) e à política de ciência, tecnologia e inovação $C \& T \& I$ (o acesso ao financiamento público).

\section{$4 \quad$ Resultados}

\subsection{Características das pequenas e médias empresas que colaboram com grupos de pesquisa de universidades e institutos públicos de pesquisa}

A categorização das empresas interativas do DGP/CNPq segundo faixas de porte de pessoal ocupado mostra um predomínio de empresas com até 19 pessoas ocupadas $(40,10 \%)$ (Tabela 01). Já as empresas interativas com 20 a 99 e 100 a 499 empregados têm, respectivamente, participações relativas de $31,00 \%$ e $28,90 \%$ na base de dados.

Tabela 01: Empresas interativas com grupos de pesquisa, segundo faixas de pessoal ocupado

\begin{tabular}{lrr}
\hline Faixas de pessoal ocupado & $\mathrm{N}^{\circ}$ de empresas & $\%$ de empresas \\
\hline até 19 pessoas ocupadas & 729 & 40,10 \\
de 20 a 99 pes soas ocupadas & 564 & 31,00 \\
de 100 a 499 pessoas ocupadas & 526 & 28,90 \\
\hline Total & 1819 & 100,00 \\
\hline
\end{tabular}

Fonte: Elaboração própria, a partir de DGP/CNPq e RAIS

A Tabela 02 mostra que o comércio e os outros serviços (isto é, todos os serviços, exceto os knowledge intensive business sectors ou KIBs) constituem os setores de atividade econômica com maior participação de empresas no conjunto de PMEs interativas com grupos de pesquisa de universidades e IPPs. Tais setores são seguidos pelos setores industriais dominados por fornecedores; KIBs; industriais baseados em ciência e intensivos em P\&D; e industriais 
fornecedores especializados ${ }^{3}$. É importante notar, todavia, que as empresas pertencentes aos KIBs correspondem a 40,10\% do total de PMEs de serviços da base de dados do DGP/CNPq. Ademais, tem-se que as PMEs dos setores industriais baseados em ciência e intensivos em P\&D representam $27,58 \%$ das empresas indústrias desta mesma base de dados.

A desagregação das informações setoriais referentes à classificação das PMEs interativas com grupos de pesquisa pelo nível de intensidade tecnológica mostra, por um lado, que é relevante a participação das empresas dos setores de atividade caracterizados pela menor intensidade tecnológica: a) setores industriais dominados por fornecedores $(22,2 \%)$, com destaque para a fabricação de produtos alimentícios $(6,1 \%)$; b) de outros serviços (que não os KIBs) $(19,8 \%)$, com destaque para as atividades associativas $(9,4 \%)$ e a produção e distribuição de eletricidade, gás e água (3.0\%); e de c) comércio (10,2\%), entre as quais as empresas mais numerosas são as de comércio por atacado, representantes comerciais e agentes do comércio $(5,7 \%)$ e de comércio varejista e reparação de objetos pessoais e domésticos $(4,1 \%)$. Ademais, é não desprezível a participação de PMEs dos setores de agricultura, pecuária, silvicultura e exploração florestal $(6,2 \%)$ no conjunto de empresas interativas.

Por outro lado, no segundo conjunto de PMEs interativas encontram-se aquelas pertencentes aos setores de atividade econômica destacados pela literatura que investiga as interações U-E por mostrarem interações mais intensas com universidades e IPPs, quais sejam, as empresas dos KIBs (HIPP; GRUPP, 2005; MILES, 2007) e dos setores industriais baseados em ciência e intensivos em P\&D (KLEVORICK et al., 1995; COHEN et al., 2002; SCHARTINGER et al., 2002; MOHNEN e HOAREAU, 2003; LAURSEN e SALTER, 2004, entre outros). De fato, destacam-se na base do DGP/CNPq as PMEs interativas dos seguintes setores de atividade possuidores de maiores oportunidades tecnológicas: 1) os KIBs $(13,2 \%)$, com destaque para os serviços prestados principalmente às empresas $(6,0 \%)$ e as atividades de informática e serviços relacionados $(4,5 \%)$; e 2$)$ os setores industriais "baseados em ciência e intensivos em P\&D" (12,0\%), entre os quais se destacam as empresas produtoras de produtos químicos (5,7\%). Sobressaem-se também as PMEs dos setores "fornecedores especializados" $(8,5 \%)$, com maior participação das fabricantes de máquinas e equipamentos $(3,7 \%)$ e dos fabricantes de instrumentos médico-hospitalares, precisão/ópticos $(2,9 \%)$.

Esses resultados corroboram as evidências de trabalhos anteriores que, utilizando os dados da PINTEC (2000) (ALBUQUERQUE et al., 2005), DGP/CNPq (CALIARI e RAPINI, 2017) e de surveys com empresas de Minas Gerais (MG Survey) (RAPINI et al., 2009) e empresas interativas com grupos de pesquisa do DGP/CNPq (BRITTO e OLIVEIRA, 2011; PINHO, 2011) encontraram que mesmo em setores de baixa e média intensidade tecnológica as universidades e os IPPs desempenham um importante papel no suporte à inovação das firmas.

\footnotetext{
3 Matos e Arroio (2011) identificam que a maior parte das micro e pequenas empresas brasileiras atua em segmentos caracterizados pelo baixo conteúdo tecnológico e maior frequência de pessoal ocupado com menores níveis de qualificação. Os segmentos tradicionalmente dominados por estas empresas são os seguintes: confecção, couro e calçados; alimentos e bebidas; comércio varejista; serviços terceirizados por outras empresas (limpeza e segurança); e serviços pessoais. Ainda segundo os autores, é também relevante a contribuição das micro e pequenas empresas em atividades consideradas intensivas em conhecimento. Nessas atividades destacam-se os serviços técnicos profissionais, como advocacia, auditoria, arquitetura, marketing e publicidade e veterinária. Os segmentos de grande dinamismo destas empresas são aqueles relacionados às tecnologias de informação e comunicação, com destaque para o desenvolvimento de softwares e conteúdos para diferentes mídias. Trata-se de serviços customizados e intensivo em conhecimento, com uma forte presença de pessoas altamente qualificadas.
} 
Tabela 02. Empresas interativas com grupos de pesquisa, segundo padrões setoriais de mudança tecnológica

\begin{tabular}{|c|c|c|}
\hline Setores & Total & $(\%)$ \\
\hline \multicolumn{3}{|l|}{ Setores dominados por fornecedores } \\
\hline Indústria extrativa & 20 & 1,10 \\
\hline Fabric. prod. alimenticios & 112 & 6,16 \\
\hline Fabric.bebidas & 14 & 0,77 \\
\hline Fabric. prod. texteis & 13 & 0,71 \\
\hline Confec. art. vestuário/acess. & 1 & 0,05 \\
\hline Couros, artef. couro e calçados & 7 & 0,38 \\
\hline Fabric. prod. madeira & 19 & 1,04 \\
\hline Fabric. papel, emb. e artef. papel & 8 & 0,44 \\
\hline Edição, impres. rep. de gravações & 1 & 0,05 \\
\hline Coque, comb. nucleares e álcool & 13 & 0,71 \\
\hline Fabric. art. borracha e plástico & 60 & 3,30 \\
\hline Fabric. prod. min. não-metálicos & 52 & 2,86 \\
\hline Fabric. prod. de metal & 59 & 3,24 \\
\hline Fabric. de artigos do mobiliário & 18 & 0,99 \\
\hline Fabric. produtos diversos & 6 & 0,33 \\
\hline & & 22,16 \\
\hline \multicolumn{3}{|l|}{ Setores fornecedores especializados } \\
\hline Fabric. celulose e out. pastas & 1 & 0,05 \\
\hline Fabric. máq. e equipamentos & 68 & 3,74 \\
\hline Fabric. mat. eletrôn. básico & 17 & 0,93 \\
\hline Instr.méd.-hosp.,precisao/ópticos & 53 & 2,91 \\
\hline Fabric. peças/acess.p/veículos & 9 & 0,49 \\
\hline Fabric. out. equip. de trans porte & 6 & 0,33 \\
\hline
\end{tabular}

Setores intensivos em economias de escala e de produção em massa

Produtos siderurgicos

Metalurg. não-ferrosos/fundição

Setores baseados na ciência e intensivos em P\&D

Fabric.produtos químicos

Fabric. produtos farmacêuticos

Fabric. máq. escrit./equip. informát.

Fabric. máq. apar. e mat. elétrico

Fabric. apar. equip. comunicaç.

Knowledge Intensive Business Services (Kibs)

Atividad. informát. e serviç. relacionad. 
Cont. Tabela 02. Empresas interativas com grupos de pesquisa, segundo padrões setoriais de mudança tecnológica

\section{Outros Serviços}

Prod. e distribuiç. eletricidad, gás e agua $\quad 54$

Alojam. e alimentaç.

Transport. terrestr.

Transport. aquaviár.

Atividad. anexas e auxiliar. dos transport. e agênc. de viagem

Atividad. imobiliár.

Aluguel veícul., máq. equip. s/ condut. ou operad. e de obj. pe

Saude e serviç. sociais

Limpez. urban. e es got. e atividad. relacionad.

Atividad. recrativ., cultur. e desportiv.

Intermediação financeira, seguros, previdência e serviços relacionados

2

Administraç. públic, defes. e seguridad. social 51

Comércio; reparação de veículos automotores, objetos pessoais e domésticos

Comérc. e reparaç. de veíc. automotor. e motocliclet.; e comérc 8

Com. Atacado, repr. comerc. e agent. do comerc 104

\begin{tabular}{lrr}
\hline & & 10,23 \\
\hline Agricultura, pecuária, silvicultura e exploração florestal & 112 & \\
\hline Pesca, aquicültura e serviços relacionados & 11 & 6,16 \\
\hline & & 0,60 \\
\hline Construção & 48 & 2,64 \\
\hline Outros & 6 & 0,33 \\
\hline Total dos setores & & 100,0 \\
\hline
\end{tabular}

Fonte: Elaboração própria, a partir de DGP/CNPq e RAIS

No que diz respeito ao número de empregados com nível superior ou mais no total de empregados, tomado como proxy da capacidade de absorção das PMEs interativas com grupos de pesquisa no Censo de 2010, as informações da RAIS indicam que $42,44 \%$ das empresas possuem $9,9 \%$ ou menos de seus empregados com esse nível de qualificação (Tabela 03). Essas empresas estão mais presentes na faixa de porte de até 19 empregados $^{4}$ (Tabela 04).

\footnotetext{
${ }^{4}$ No conjunto de PMEs interativas, 367 empresas, ou 20,17\% do total das empresas não possuem recursos humanos com nível superior ou mais. Essas empresas se concentram na faixa de até 19 empregados (ou seja, 80,11\% das empresas que não possuem empregados com nível superior ou mais pertencem a essa faixa de porte).
} 
Adicionalmente, tem-se que $29,14 \%$ das PMEs interativas possuem de 10 a $39,9 \%$ dos seus empregados com nível superior ou mais e que 4,9\% das empresas apresentam entre 90 e $100 \%$ de seus recursos humanos com esse nível de formação educacional. É interessante notar que as empresas com 90 a $100 \%$ de seus recursos humanos com nível superior ou mais também estão mais presentes na faixa de porte de empresas com até 19 empregados.

Os dados da RAIS sugerem, por um lado, que parcela significativa das PMEs interativas possui escassos recursos internos em termos de recursos humanos qualificados, que se traduzem em capacidade de absorção. A capacidade de absorção é apontada pela literatura como uma pré-condição para a cooperação com universidades e IPPs. Por outro lado, os dados da RAIS podem sinalizar também que tais empresas, ao possuírem menos recursos internos necessitam significativamente de conhecimento externo, adquirido por meio de processos cooperativos com grupos de pesquisa de universidades e IPPs.

Tabela 03. Empresas interativas com grupos de pesquisa, segundo faixas de empregados com nível superior ou mais

\begin{tabular}{lrr}
\hline Empregados com Ensino Superior ou mais no total de empregados (\%) & Empresas & Empresas (\%) \\
\hline $90-100$ & 90 & 4,95 \\
$60-89,9$ & 216 & 11,87 \\
$40-59,9$ & 211 & 11,60 \\
$10-39,9$ & 530 & 29,14 \\
$\leq 9,9$ & 772 & 42,44 \\
\hline Total & 1819 & 100,00 \\
\hline
\end{tabular}

Fonte: Elaboração própria, a partir de DGP/CNPq e RAIS

Tabela 04. Empregados com nível superior ou mais por faixas de porte das empresas interativas com grupos de pesquisa

\begin{tabular}{lrrrrrrrrrr}
\hline Faixas de pessoal ocupado & $\leq 9,9$ & $(\%)$ & $10-39,9$ & $(\%)$ & $40-59,9$ & $(\%)$ & 60 a 89,9 & $(\%)$ & $90-100$ & $(\%)$ \\
\hline até 19 pessoas ocupadas & 329 & 42,62 & 167 & 31,51 & 85 & 40,28 & 78 & 36,11 & 70 & 77,78 \\
de 20 a 99 pessoas ocupadas & 239 & 30,96 & 189 & 35,66 & 55 & 26,07 & 70 & 32,41 & 11 & 12,22 \\
de 100 a 499 pessoas ocupadas & 204 & 26,42 & 174 & 32,83 & 71 & 33,65 & 68 & 31,48 & 9 & 10,00 \\
\hline Total & 772 & 100,00 & 530 & 100,00 & 211 & 100,00 & 216 & 100,00 & 90 & 100,00 \\
\hline
\end{tabular}

Fonte: Elaboração própria, a partir de DGP/CNPq e RAIS

No que tange à interação anterior, tomada como proxy da experiência prévia da firma com a cooperação U-E, os dados do DGP/CNPq apontam que que 56,62\% das empresas interagiram no Censo anterior (Censo 2008). Esse seria um indício que de que a firma possui ou está construindo capacitações (capabilities) para o estabelecimento do relacionamento cooperativo U-E, posto que já interagiu com grupos de pesquisa de universidades e IPPs. Já 43,38\% das PMEs investigadas não se engajaram, no Censo 2008, em relacionamentos cooperativos com grupos de pesquisa destas organizações do sistema nacional de inovação.

As informações referentes ao financiamento público apontam que uma parcela minoritária (16,22\%) de PMEs do DGP/CNPq (Censo 2010) recebeu algum tipo de financiamento público no período analisado. Esse seria um indicativo de que os instrumentos recentes da política de $\mathrm{C} \& \mathrm{~T} \& \mathrm{I}$ tiveram um alcance limitado junto a essas empresas. Todavia, é 
importante notar que, dada à limitação de informações no âmbito deste trabalho, é provável que o acesso das empresas do DGP/CNPq ao financiamento público esteja subestimado.

Quando tais informações são desagregadas por faixas de porte das empresas interativas, há, por um lado, evidências de que aquelas com 100 a 499 empregados possuem menor participação relativa na base do DGP/CNPq $(28,90 \%$ do total de PMEs). Contudo, elas constituem a faixa de porte que mais acessou o financiamento público (40,00\% das empresas que tiveram financiamento público). Por outro lado, embora as empresas com até 19 empregados sejam as mais representadas na base de dados (40,10\% do total de PMEs), constatase que essa faixa acessou menos o financiamento público do que as empresas da faixa de porte superior. Isto é, as empresas com até 19 empregados constituem 33,90\% das empresas que receberam financiamento público.

No que tange ao tipo de instrumento da política de C\&T\&I, os dados da Tabela 05 sugerem que as empresas das faixas de porte de 20 a 99 e 100 a 499 empregados acessaram programas públicos de apoio direto mais diversificados do que as empresas com até 19 empregados. As PMEs dessa última faixa de porte acessaram recursos oriundos principalmente de Subvenção (FINEP), PIPE (FAPESP) e RHAE Pesquisador na Empresa (CNPq). Ademais dos instrumentos da FINEP, FAPESP e CNPq, algumas empresas das faixas de 20 a 99 e 100 a 499 acessaram também instrumentos do BNDES e do MCTI.

Tabela 05. Empresas interativas com grupos de pesquisa que acessaram o financiamento público por faixa de porte e instrumento da política de C\&T\&I

\begin{tabular}{clccc}
\hline & $\mathbf{( 0 , 1 9 ]}$ & $\mathbf{( 1 9 , 9 9 ]}$ & $\mathbf{( 9 9 , 4 9 9 ]}$ \\
\hline \multirow{3}{*}{ FINEP } & Não-Reembolsável & 13 & 15 & 30 \\
& Reembolsável & 10 & 18 & 32 \\
& Subvenção & 40 & 34 & 26 \\
& BNDES Profarma & & & 2 \\
& BNDES Funtec & 1 & \\
& BNDES Prosoft & & & 3 \\
& PSI - Inovação Tecnologica & & 1 & 3 \\
& Inovação Produção & 1 & 3 \\
& Capital Inovador & & & 1 \\
& Inovação Tecnológica & & 1 & 1 \\
& Profarma Produção & & & 1 \\
BNDES & Outros & & & 3 \\
& Mercado de Capitais & 5 & 3 & 2 \\
FAPESP & PIPE & 28 & 12 & 4 \\
CNPq & RHAE Pesquisador na Empresa & 25 & 9 & 2 \\
MCTI & MCTI & 6 & 18 & 62 \\
\hline
\end{tabular}

Fonte: Elaboração própria, a partir de CNPq RAIS, FINEP, BNDES, MCTI e FAPESP

\subsection{Características dos relacionamentos cooperativos de pequenas e medias empresas $e$} grupos de pesquisa de universidades de institutos públicos de pesquisa

Em sua maioria, as empresas da base do DGP/CNPq possuem até duas interações, totalizando 95,27\% destas. Dessa forma, são pouco numerosas as PMEs que estão envolvidas em um número maior de relacionamentos cooperativos com universidades e IPPs. Os modos de interação mais frequentes nas interações entre grupos de pesquisa de universidades e IPPs e 
PMEs são os de tipo bidirecional (67,64\%) (incluem os tipos de relacionamento "Pesquisa científica com considerações de uso imediato dos resultados" e "Pesquisa científica sem considerações de uso imediato dos resultados") (Tabela 06). Outros 32,6\% são relacionamentos unidirecionais (incluem transferência de tecnologia, desenvolvimento de software, engenharia não rotineira, consultoria, treinamento e fornecimento de insumos materiais). Esses dados indicam que as colaborações entre os grupos de pesquisa e o setor produtivo, conforme indicado pelos líderes, envolvem não apenas um fluxo unidirecional, mas também bidirecional de informação e conhecimento, em que podem ser observados fluxos de conhecimento da universidade para as empresas e das empresas para a universidade.

Os modos de interação mais frequentes, que partem dos grupos de pesquisa para o setor produtivo, são pesquisa científica com uso imediato dos resultados (podendo-se considerar como pesquisa de curto prazo), com cerca de $45,17 \%$ do total dos relacionamentos, pesquisa científica sem uso imediato dos resultados (podendo-se considerar pesquisa de longo prazo) $(22,47 \%)$ e transferência de tecnologia $(10,86 \%)$. Esses três tipos de relacionamento abarcam $78,50 \%$ do total. Por sua vez, entre os relacionamentos que partem do setor produtivo para os grupos de pesquisa, ainda que em menor magnitude, os mais frequentes foram insumos materiais $(4,87 \%)$ e engenharia não rotineira $(3,53 \%)^{5}$.

Tabela 06. Tipos de relacionamento entre grupos de pesquisa e empresas, segundo tipos de relacionamento

\begin{tabular}{lrr}
\hline Modos de interação de acordo com a origem & Numero & \% \\
\hline Grupos de Pes quisa-> Setor produtivo & 149 & 6,92 \\
Consultoria tecnica & 34 & 1,58 \\
Engenharia não rotineira (a) & 24 & 1,11 \\
Desenvolvimento de software & 973 & 45,17 \\
Pesquis a científica com uso imediato & 484 & 22,47 \\
Pesquis a científica sem uso imediato & 234 & 10,86 \\
Transferência de tecnologia & 34 & 1,58 \\
Treinamento de pes soal & 7 & 0,32 \\
Insumos materiais & & \\
Setor produtivo-> Grupos de Pes quisa & 76 & 3,53 \\
Engenharia não rotineira (b) & 10 & 0,46 \\
Desenvolvimento de software & 20 & 0,93 \\
Trans ferência de tecnologia & 4 & 0,19 \\
Treinamento de pes oal & 105 & 4,87 \\
Insumos materiais & 2154 & 100,00 \\
\hline Total de relacionamentos
\end{tabular}

Fonte: Elaboração própria, a partir de DGP/CNPq e RAIS

\subsection{Síntese dos resultados}

A Tabela 07 apresenta as estatísticas descritivas referentes às características acima referidas. Em média, as PMEs investigadas possuem 1,2727 interações, sendo o desvio padrão de 0,7831 (e variância igual a 0,6132). Os dados do DGP/CNPq mostram, portanto que é bastante baixa a intensidade dos relacionamentos U-E de PMEs brasileiras. Note-se que esses

\footnotetext{
${ }^{5}$ Esse resultado é coerente com resultados prévios (por exemplo, BRITTO e OLIVEIRA, 2011; ARZA et al., 2015) que mostraram que as principais recompensas da interação de empresas brasileiras com organizações públicas de pesquisa relacionavam-se à abertura de opções para resolver problemas de produção de curto prazo, em vez de aprimorarem sua performance inovativa de longo prazo). Nesse caso, a possibilidade de realizar testes e usar recursos de organizações públicas de pesquisa estiveram entre as mais importantes recompensas dos relacionamentos cooperativos U-E, conforme as empresas.
} 
resultados são convergentes com os resultados de Santoro (2000) no contexto de relacionamentos U-E de centros de pesquisa universitários dos Estados Unidos. Pode-se observar também que as PMEs analisadas são muito heterogêneas, particularmente em relação às variáveis número de empregados e número de empregados com nível superior ou mais.

Tabela 07: Resumo dos dados das empresas que possuem algum tipo de relacionamento com grupos de pesquisa

\begin{tabular}{|c|c|c|c|c|c|c|c|c|}
\hline Variável & Mínimo & $1^{a}$ quartil & Média & Mediana & $3^{\mathrm{a}}$ quartil & Máximo & $\begin{array}{l}\text { Desvio } \\
\text { padrão }\end{array}$ & $\begin{array}{c}\text { Coeficiente de } \\
\text { variação de } \\
\text { Pearson }(\%) \\
\end{array}$ \\
\hline $\begin{array}{l}\mathrm{N}^{\circ} \text { de interações com } \\
\text { grupos de pesquisa }\end{array}$ & 1 & 1 & 1,2727 & 1 & 1 & 13 & 0,7831 & 61,53 \\
\hline $\mathrm{N}^{\mathrm{o}}$ de empregados & 1 & 9 & 86,7559 & 33 & 122 & 495 & 113,3075 & 130,6 \\
\hline $\begin{array}{l}\text { No de empregados com } \\
\text { nível superior ou pós- } \\
\text { graduação }\end{array}$ & 0 & 1 & 22,6504 & 5 & 20 & 355 & 46,281 & 204,33 \\
\hline $\begin{array}{l}\text { Percentual de } \\
\text { empregados com nível } \\
\text { superior ou pós- } \\
\text { graduação }\end{array}$ & 0 & 2,91 & 26,7547 & 14,29 & 44,54 & 100 & 29,3893 & 109,85 \\
\hline $\begin{array}{l}\mathrm{N}^{\mathrm{o}} \text { de relações } \\
\text { bidirecionais }\end{array}$ & 0 & 0 & 0,7982 & 1 & 1 & 10 & 0,7922 & 99,24 \\
\hline $\begin{array}{l}\text { Percentual de relações } \\
\text { bidirecionais }\end{array}$ & 0 & 0 & 61,5832 & 100 & 100 & 100 & 46,6811 & 75,8 \\
\hline
\end{tabular}

Fonte: Elaboração própria, a partir do DGP/CNPq e da RAIS

\section{$5 \quad$ Considerações finais}

As estatísticas descritivas apresentadas neste trabalho evidenciam que o nível de intensidade das interações U-E de PMEs brasileiras é baixo (média=1,2727), sendo que 95,27\% das empresas da base do DGP/CNPq possuem até duas interações. Os dados do DGP/CNPq mostram também que os tipos de relacionamento mais frequentes nas interações entre grupos de pesquisa de universidades e IPPs e PMEs são os de tipo bidirecional $(67,64 \%)$. Esse trabalho evidencia ainda que não há, entre as PMEs brasileiras, uma clara divisão entre setores de alta e baixa tecnologia com respeito ao engajamento na interação U-E.

Embora as PMEs investigadas sejam relativamente homogêneas em relação à intensidade da interação U-E, constata-se que essas empresas são bastante heterogêneas, particularmente em relação ao número de empregados e número de empregados com nível superior ou mais (proxy adotada para a capacidade de absorção da empresa).

Os resultados deste trabalho traduzem-se em implicações de políticas. É importante que as políticas de apoio à inovação em PMEs considerem o papel dos direcionadores da colaboração entre essas firmas e universidades e IPPs. As políticas devem incorporar a necessidade de elevação da capacidade de absorção de PMEs. Firmas que possuem capacitações internas mais desenvolvidas conseguem delimitar melhor suas demandas e a realizar uma busca mais acurada de soluções para os seus problemas (independentemente da localização geográfica da universidade que pode colaborar na solução do problema enfrentado pela firma) (GARCIA et al., 2014). 
O fomento da capacidade de absorção de PMEs brasileiras pode ser implementado pelas vias dos estímulos (1) à contratação e fixação de recursos humanos qualificados (graduados e, particularmente, pós-graduados) nas firmas; e (2) aos esforços de P\&D por meio de instrumentos voltados às particularidades dessas firmas.

Este trabalho apresenta evidências de que os instrumentos recentes da política de $\mathrm{C} \& \mathrm{~T} \& \mathrm{I}$ tiveram um alcance limitado junto às empresas do DGP/CNPq. O apoio financeiro a projetos de pesquisa também parece oportuno, uma vez que propicia o desenvolvimento da capacidade de absorção de PMEs e favorece o aumento da intensidade da interação U-E e o estabelecimento de relacionamentos bidirecionais entre grupos de pesquisa e essas empresas (OLIVEIRA, 2019). Adicionalmente, dado que os benefícios de longo prazo da colaboração são maiores do que os benefícios de curto prazo (GARCIA et al, 2019) e que a experiência acumulada pela firma no estabelecimento de relacionamentos cooperativos é conducente à intensidade da colaboração interorganizacional (OLIVEIRA, 2019), é importante que as políticas considerem também a adoção de instrumentos que encorajem a manutenção de relações ao longo do tempo, especialmente através de programas que considerem a duração da colaboração U-E. Dessa maneira, propiciar-se-á o acúmulo de experiências de PMEs com relações interorganizacionais e o consequente desenvolvimento da capacitação (capability) da firma requerida para interagir efetivamente com universidades e IPPs, seja com o mesmo parceiro através de períodos de tempo recorrentes, ou com parceiros diferentes.

\section{Referencias}

ALBUQUERQUE, E. SILVA, L.; POVOA, L. Diferenciação intersetorial na interação entre empresas e universidades no Brasil. São Paulo em Perspectiva, v. 19, n. 1, p. 95-104,2005.

ARZA, V. et. al. Channels and benefits of interactions between public research organizations and industry: comparing country cases in Africa, Asia, and Latin America; In ALBUQUERQUE, E. et. al. (Eds) Developing National Systems of Innovation: UniversityIndustry Interactions in the Global South. Cheltenham/Ottawa: Edward Elgar Publishing, 2015, p. 164-193.

BASTOS, C.; BRITTO, J. Inovação e geração de conhecimento científico e tecnológico no Brasil: uma análise dos dados de cooperação da Pintec segundo porte e origem de capital. Revista Brasileira de Inovação, 16 (1), p. 35-62, 2017.

BRITTO, J.; OLIVEIRA, B. Padrões setoriais de interação universidade-empresa no Brasil: um mapeamento de competências a partir de informações da pesquisa "Brazil Survey". Revista de Economia, v. 37, n. especial, p. 167-212, 2011.

BRUNEEL, J., D'ESTE; P., SALTER, A. Investigating the factors that diminish the barriers to university-industry collaboration. Research Policy, v. 39, n. 7, p. 858-868, 2010.

CALIARI, T.; RAPINI, M. Diferenciais da distância geográfica na interação universidadeempresa no Brasil: um foco sobre as características dos agentes e das interações. Nova Economia, v.27, n.1, p.271-302, 2017.

CARDAMONE, P.; PUPO, V. R\&D cooperation between firms and universities: some evidence in five European countries. Working Paper n. 01-15. Università Della Calabria, 2015. CARRIJO, M.; BOTELHO, M. Cooperação e inovação: uma análise dos resultados do Programa de Apoio à Pesquisa em Empresas (Pappe). Revista Brasileira de Inovação, v. 12, n. 2, p. 417-448, 2013. 
CHIARINI, T.; RAPINI, M.; OLIVEIRA, V. Obstáculos à inovação e porte das empresas industriais. Rumo a políticas públicas de incentivo à inovação mais assertivas no Brasil. $4^{o}$ Encontro Nacional de Economia Industrial e Inovação. Campinas: ABEIN, 2019.

COHEN, W.; LEVINTHAL, D. Absorptive Capacity: A New Perspective on Learning and Innovation. Administrative Science Quarterly, v. 35, p. 128-1S2, 1990.

COHEN, W.; NELSON, R.; WALSH, J. Links and impacts: the influence of public research on industrial R\&D. Management Science, vol. 48, n.1, p, 1-23, 2002.

CYERT, R.; GOODMAN, P. Creating effective university-industry alliances: an organizational learning perspective. Organizational Dynamics, v. 25, n. 4, p. 45-57, 1997.

DE FUENTES, C.; DUTRENIT, G. Best channels of academia-industry interaction for longterm benefit. Research Policy, v. 41, n. 9, p. 1666-1682, 2012.

DREJER, I.; ØSTERGAARD, C. Exploring determinants of firms' collaboration with specific universities: Employeedriven relations and geographical proximity Regional Studies, $/ 2017$.

FREEL, M. Barriers to product innovation in small manufacturing firms. International Small Business Journal, v. 18, n. 2, p. 60-80, 2000.

FONTANA, R.; GEUNA, A.; MATT, M. Firm size and openness: the driving forces of universityindustry collaboration. SPRU Working Paper Series, n.103, 2003.

GARCIA, R. et al. Efeitos da qualidade da pesquisa acadêmica sobre a distância geográfica das interações universidade-empresa. Estudos Econômicos, v. 44, n. 1, p. 105-132, 2014.

GARCIA, R. et al. How long-term university-industry collaboration shapes the academic productivity of research groups. Innovation: Management, Policy \& Practice, v. 21, p. 1-15, 2019.

HIPP, C.; GRUPP, H. Innovation in the service sector: The demand for service-specific innovation measurement concepts and typologies. Research Policy, v. 34, p. 517-535, 2005.

KLEVORICK, A et al. On the sources and significance of inter-industry differences in technological opportunities. Research Policy, v. 24, p. 185-205, 1995.

LAURSEN, K.; SALTER, A. Searching high and low: what types of firms use universities as a source of innovation? Research Policy, v. 33, n. 8, p. 1201-1215, 2004.

LAURSEN, K.; REICHSTEIN, T.; SALTERS, A. Exploring the effect of Geographical Proximity and University Quality on University-Industry Collaboration in the United Kingdom. Regional Studies, v. 45, n. 4, p. 507-523, 2011.

MATOS, M.; ARROIO, A. Políticas de apoio a micro e pequenas empresas no Brasil: avanços no período recente e perspectivas futuras. Santiago do Chile: CEPAL, 2011.

MILES, I. Research and development (R\&D) beyond manufacturing: the strange case of services R\&D. R\&D Management, v. 37, n. 3, p.249-268 2007.

MOHNEN, P.; HOAREAU, C. What type of enterprise forges close links with universities and government labs? Evidence from CIS 2. Managerial and Decision Economics, v. 24, n. 2-3, p. 133-145, 2003.

MOLINA-YCAZA, D.; SÁNCHEZ-RIOFRÍO, A. Obstáculos para la micro, pequeña y mediana empresa en América Latina Revista Pymes, Innovación y Desarrollo, v. 4, n.2, p. 2136, 2016.

OLIVEIRA, V. Relacionamentos cooperativos entre pequenas e médias empresas brasileiras $e$ universidades e institutos públicos de pesquisa: uma investigação sobre os fatores 
direcionadores (drivers) da interação e dos tipos de relacionamentos. Tese (Doutorado). Campinas: IE/UNICAMP, 2019.

PINHO, M. A visão das empresas sobre as relações entre universidade e empresa no Brasil: uma análise baseada nas categorias de intensidade tecnológica. Revista de Economia, v. 37, n. especial, p. 279-306, 2011.

RAPINI, M.; OLIVEIRA, V.; CALLIARI, T. Como a interação universidade-empresa é remunerada no Brasil: evidências dos grupos de pesquisa do CNPq. Revista Brasileira de Inovação, v. 15, n. 2, p. 219-246, 2016.

RASIAH, R; GOVINDARAJU, C. University-industry R\&D collaboration in the automotive, biotechnology and electronic firms in Malaysia. Seoul Journal of Economics, v. 22, n. 4, p. 530-550, 2009.

RIGHI, H.; RAPINI, M. Metodologia e apresentação da Base de Dados do Censo 2004 do Diretório dos Grupos de Pesquisa do Conselho Nacional de Pesquisa Científica e Tecnológica (CNPq). In: SUZIGAN, W.; ALBUQUERQUE, E.; CARIO, S. (Orgs) Em busca da inovação: interação universidade-empresa no Brasil. Belo Horizonte: Editora Autêntica, 2011, p. 45-73.

ROSA, A.; RUFFONI, J.; GARCIA, R. Capacidade de absorção e desempenho inovativo: uma análise para as firmas interativas com grupos de pesquisa universitários das áreas das engenharias do Rio Grande do Sul. Revista Brasileira de Economia de Empresas, v. 18, n. 1, p. 71-91, 2018.

ROTHWELL, R. Small firms, innovation and industrial change. Small Business Economics, v. 1, n. 1, p 51-64, 1989.

ROVERE, R. Perspectivas das Micro, Pequenas e Médias Empresas no Brasil. Revista de Economia Contemporânea, Rio de Janeiro, v. 5, n.Ed. Espec., p. 20-38, 2001.

SANTORO, M. Success breeds success: the linkage between relationship intensity and tangible outcomes in industry- university collaborative ventures. The Journal of High Technology Management Research, v. 11, n. 2, p. 255-273, 2000.

SANTORO, M.; CHAKRABARTI, A. Firm size and technology centrality in industryuniversity interactions. Research Policy, v. 31, p.1163-1180, 2002.

SCHARTINGER, D. et al. Knowledge interactions between universities and industry in Austria: sectoral patterns and determinants. Research Policy, v. 31, p. 303-328, 2002.

SERVIÇO BRASILEIRO DE APOIO ÀS MICRO E PEQUENAS EMPRESAS. Anuário do trabalho na micro e pequena empresa. 6. ed. Brasília: DIEESE, 2013.

SUZIGAN, W. et al. University and industry linkages in Brazil: some preliminary and descriptive results. Seoul Journal of Economics, v. 22, n. 4, p. 591-691, 2009.

TEIXEIRA, A. L. et al. Dimensões da capacidade de absorção, qualificação da mão de obra, P\&D e desempenho inovativo. Revista Brasileira de Inovação, v. 15, n. 1, p. 139-164, 2016.

TORRES, A. et al What are the factors driving university-industry linkages in latecomer firms: evidence from Mexico. Sciecnce and Public Policy, v. 36, n. 1, p. 31-42, 2011.

TORRES, P. H.; BOTELHO, M. Financiamento à inovação e interação entre atividades científicas e tecnológicas: uma análise do Pappe. Revista Brasileira de Inovação, v. 17, n. 1, p. 89-118, 2018.

ZEVALLOS, E. Micro, pequenas y medianas empresas en América Latina Revista de la Cepal. v. 7, n. 9, p. 53-70, 2003. 